\title{
Analysis of a Human-Machine Interface for Cooperative Truck Overtaking Maneuvers on Freeways: Increase Success Rate and Assess Driving Behavior during System Failures
}

\author{
Jana Fank *D, Christian Knies and Frank Diermeyer (D)
}

Citation: Fank, J.; Knies, C.; Diermeyer, F. Analysis of a Human-Machine Interface for Cooperative Truck Overtaking Maneuvers on Freeways: Increase Success Rate and Assess Driving Behavior during System Failures. Multimodal Technol. Interact. 2021, 5, 69. https://doi.org/10.3390/ mti5110069

Academic Editors: Mu-Chun Su and Cristina Portalés Ricart

Received: 14 September 2021

Accepted: 28 October 2021

Published: 2 November 2021

Publisher's Note: MDPI stays neutral with regard to jurisdictional claims in published maps and institutional affiliations.

Copyright: (c) 2021 by the authors. Licensee MDPI, Basel, Switzerland. This article is an open access article distributed under the terms and conditions of the Creative Commons Attribution (CC BY) license (https:// creativecommons.org/licenses/by/ $4.0 /)$.
Institute of Automotive Technology, Technical University of Munich, 85748 Garching, Germany; christian.knies@tum.de (C.K.); diermeyer@ftm.mw.tum.de (F.D.)

* Correspondence: fank@ftm.mw.tum.de; Tel.: +49-89-289-15779

\begin{abstract}
Cooperation between road users based on V2X communication has the potential to make road traffic safer and more efficient. The exchange of information enables the cooperative orchestration of critical traffic situations, such as truck overtaking maneuvers on freeways. With the benefit of such a system, questions arise concerning system failure or the abrupt and unexpected behavior of road users. A human-machine interface (HMI) organizes and negotiates the cooperation between drivers and maintains smooth interaction, trust, and system acceptance, even in the case of a possible system failure. A study was conducted with 30 truck drivers on a dynamic truck driving simulator to analyze the negotiation of cooperation requests and the reaction of truck drivers to potential system failures. The results show that an automated cooperation request does not translate into a significantly higher cooperation success rate. System failures in cooperative truck passing maneuvers are not considered critical by truck drivers in this simulated environment. The next step in the development process is to investigate how the success rate of truck overtaking maneuvers on freeways can be further increased as well as the implementation of the system in a real vehicle to investigate the reaction behavior of truck drivers in case of system failures in a real environment.
\end{abstract}

Keywords: human-machine interaction; truck drivers; cooperative driving; V2X communication; driving simulator study; system failure

\section{Introduction}

Cooperation on the road is an essential part of efficient and conflict-free traffic [1,2]. Cooperation is defined as jointly working towards a common goal [3]. Regarding traffic, this means working together to successfully complete a traffic maneuver [4]. As an example, by changing lanes or reducing speed the driver can create a gap in the traffic flow to help his or her partner to merge onto the freeway [2]. With the implementation of data-sharing possibilities provided by Vehicle-2-X (V2X) communication, cooperation can be made more successful, efficient, and safe. More traffic situations can be improved for all road users where there has been little or barely any cooperation before. One of these situations is the truck overtaking maneuver on freeways. Blocking the lane by overtaking trucks is the most frequent traffic conflict for car drivers [5]. The sudden lane change in the overtaking truck is also the most threatening truck driving maneuver on freeways [5]. In addition to the subjectively perceived problems, observations and driving simulator studies show that just prior to a truck overtaking maneuver, truck drivers often tailgate the truck in front [5-7]. Due to a small speed difference between the overtaking trucks, the maneuvers can stretch for a long time and result in a significant disturbance of overall traffic; Germany, for example, therefore passed legislation to limit these maneuvers to $45 \mathrm{~s}[6,7]$. The increasing demand and time pressure on truck drivers worsens this issue [8] since any vehicle ahead that is even slightly slower becomes a serious risk to their schedule, which in turn leads to more overtaking maneuvers. Collective perception based on V2X communication makes it 
possible to coordinate such critical maneuvers in the future. The ideal overtaking moment, speed, and distance between the vehicles are negotiated between all involved parties, and the cooperative maneuver is safeguarded.

Within 10 steps, the maneuver is planned and executed (see Table 1) [8]. It is important that the relevant steps are transmitted to and displayed by a suitable HMI (see Table 1) [9] so that the driver can perform the cooperative overtaking maneuver successfully. Truck drivers desire participation in the negotiation process for cooperation in truck overtaking maneuvers on freeways $[6,9]$. Submitting a cooperation request from the overtaker perspective (overtaker) marks the beginning of the cooperation negotiation. Accepting the request from the overtaken party (overtaken) finalizes the negotiation and initiates the actual overtaking maneuver. From this point on, the partners have agreed to follow the negotiated arrangements. Sending as well as accepting a request can be performed manually by the driver himself or automated by the system. From the perspective of the overtaken, it was shown that a manual approval by the driver is acceptable and, consequently, this can lead to a high cooperation rate [6,9]. However, from the perspective of the overtaker, this high cooperation rate could not be found [9]. The question arises of whether an automated approval of the cooperation request is acceptable and leads to more frequent cooperative truck overtaking maneuvers on freeways.

Table 1. Description of the ten states of cooperative truck overtaking maneuvers on freeways (according to [8]), with the corresponding information displayed by HMI (according to [9]).

\begin{tabular}{|c|c|c|c|c|c|}
\hline \multirow{2}{*}{ No. } & \multirow{2}{*}{ State } & \multicolumn{4}{|c|}{ Information Displayed by the HMI } \\
\hline & & Status & Instruction & Request & Partner \\
\hline 1 & $\begin{array}{c}\text { Solo } \\
\text { No synchronization between } \\
\text { vehicles }\end{array}$ & & & & \multirow{10}{*}{$\begin{array}{l}\text { Position, velocity, } \\
\text { weight, engine }\end{array}$} \\
\hline 2 & $\begin{array}{c}\text { Initialization } \\
\text { Roles }(\mathrm{A})^{1} \text { and }(\mathrm{B})^{1} \text { are } \\
\text { assigned to the two trucks }\end{array}$ & (A) Searching & & & \\
\hline 3 & $\begin{array}{l}\text { Planning } \\
\text { (A) and (B) plan the } \\
\text { overtaking maneuver together }\end{array}$ & $\begin{array}{c}\text { (A) Initialized } \\
\text { (A) and (B) Cooperation } \\
\text { request }\end{array}$ & & $\begin{array}{l}\text { (A) Send } \\
\text { Request?(B) } \\
\text { Cooperate? }\end{array}$ & \\
\hline 4 & $\begin{array}{l}\text { Approach } \\
\text { (A) adjusts speed until } \\
\text { distance }<60 \mathrm{~m}\end{array}$ & $\begin{array}{l}\text { (A) and (B) Cooperation } \\
\text { confirmed }\end{array}$ & & & \\
\hline 5 & $\begin{array}{l}\text { Secure Gap (pre) } \\
\text { (A) reduces distance and } \\
\text { reacts to brake signals from } \\
\text { (B) }\end{array}$ & $\begin{array}{l}\text { (A) Lane change in } x y \\
\text { meters }\end{array}$ & & & \\
\hline 6 & $\begin{array}{l}\text { Lane Change (to 2) } \\
\text { (A) changes lane }\end{array}$ & \multicolumn{3}{|c|}{ (A) Change lane! } & \\
\hline 7 & $\begin{array}{l}\text { Pass(A) and (B) adjust their } \\
\text { speeds }\end{array}$ & $\begin{array}{l}\text { (A) Lane change in xy } \\
\text { meters } \\
\text { (A) and (B) Adjust speed }\end{array}$ & & & \\
\hline 8 & $\begin{array}{l}\text { Lane Change (to } 1 \text { ) } \\
\text { (A) changes lane }\end{array}$ & & (A) Change lane! & & \\
\hline 9 & $\begin{array}{l}\text { Secure Gap (post) } \\
\text { (B) reacts to brake signals } \\
\text { from (A) until distance is } \\
>50 \mathrm{~m}\end{array}$ & & & & \\
\hline 10 & $\begin{array}{l}\text { End } \\
\text { synchronization is resolved }\end{array}$ & $\begin{array}{l}\text { (A) and (B) Cooperation } \\
\text { complete }\end{array}$ & & & \\
\hline
\end{tabular}


In parallel to the complexity, the error-proneness of such an Advanced Driving Assistance System (ADAS) increases: the communication between vehicles can fail, vehicles without such a system can interfere with the cooperation, or the cooperating partner does not cooperate as negotiated. In an initial investigation, how truck drivers behave in such situations, how critical they perceive it, and which information they would like to receive from the system must be investigated.

This work investigates how the success rate of cooperative truck overtaking maneuvers on freeways may be increased. In addition to the development of an HMI according to current usability design paradigms, it is investigated whether the automatic submission of a cooperation request is acceptable for drivers and, as a consequence, whether the cooperation rate can be increased, especially from the overtaker's perspective. Furthermore, how truck drivers behave when a cooperative ADAS fails is investigated. For this purpose, the HMI was extended to inform the driver about incidents without overwhelming them with extensive information (see Section 4). To test the HMI, a study was conducted with 30 truck drivers on a dynamic truck driving simulator (see Section 5). The study was divided into two sections: In the first part, the truck drivers' participation in cooperation requests together with the usability of the developed HMI were investigated. In the second part, truck drivers evaluated the criticality and their trust and acceptance towards the cooperative ADAS (see Section 6) within three simulated system failure test situations (cooperator not behaving as planned, system collapse, and unplanned behavior of surrounding traffic), in relation to different information content displayed by the developed HMI. In addition, the driving behavior was recorded and analyzed.

\section{Related Work}

\subsection{Challenges of HMI Design for Cooperative Truck Overtaking Maneuvers on Freeways}

A main challenge in HMI design for a cooperative ADAS is to appeal to all cooperating partners in a negotiation maneuver [10]. In order for truck drivers to accept the cooperative ADAS, the HMI must be easy to use, but also convince the driver of its benefits [11]. In the truck overtaking scenario, as described above, cooperation emerges regardless of whether the driver is in the position of the overtaker or in the position of the overtaken [9]. The overtaken person cooperates with the overtaker by adjusting the vehicles speed. Although the overtaker is the one initiating the cooperation, the maneuver is not independent from other factors, such as the rear traffic or the current road topology [9]. Observations and studies on the cooperative behavior of truck drivers during overtaking maneuvers on freeways without a cooperative ADAS show that this consideration is not always given [6,7]. Even with high differential speeds to the oncoming traffic, as well as a high traffic volume in general, truck drivers do not cooperate with the rear traffic and thus are not reluctant to overtake [6,7]. An HMI for cooperative truck overtaking maneuvers must convince the truck driver to overtake only if it is reasonable for all road users affected. For this purpose, instructions for the driver must be presented as comprehensibly as possible [12,13]. An HMI for cooperative truck overtaking maneuvers developed according to the user-centered approach [9] showed its weaknesses precisely in the critical perspective of the overtaking driver. In the evaluation of the HMI [9], only one third of all overtaking maneuvers were performed successfully. Subjects either did not send a request to cooperate or did not follow the request to change lanes at a predetermined moment [9]. According to the authors of this study, one reason for the low cooperation rate from the perspective of the overtaker [9] was poor usability of the HMI. As stated by the participants of the study, the information content was considered too high, and the instructions were not clearly formulated [9]. In this work, an HMI is developed that attempts to minimize the information content to the necessary and provide precise instructions to the driver by applying common design paradigms. In addition, to what extent an automated submission of the cooperation request can simplify the negotiation and lead to a higher cooperation rate is investigated. 


\subsection{Dealing with System Failures during Cooperative Truck Overtaking Maneuvers on Freeways}

Previous research evaluating the HMI for cooperative truck overtaking maneuvers on freeways assumes a working system under ideal conditions $[6,9,14]$, neglecting the fact that the system has its limits [15]. Communication may be unstable or break down entirely and lead to system failure. With the final cooperation decision made by the driver (SAE-Levels 0 and 1), cooperation partners could always interrupt the planned maneuver and behave in an unplanned manner. Mixed traffic (some vehicles are equipped with the described cooperation system, others not) poses an additional challenge. Therefore, negotiated maneuvers may be hindered by other vehicles equipped with V2X communication. The criticality and the extent to which safety is compromised in the event of a system failure is the key question of this investigation. The driver should always be able to maintain control of the driving situation [16]. In particular, adequate trust that the truck driver holds in the system and how trust is affected in the case of system failures play a central role in its safe use [17]. In these situations, the task of the HMI is to inform the driver about the situation in order to avoid uncertainty or mistrust $[15,18]$. However, HMI should not increase the mental load of the driver with detailed and extensive information [19]. In a simulator study of car driver cooperation in merging and intersection situations, it was shown that explanations were helpful to drivers but did not change their acceptance of the system [15]. However, since previous studies have shown that design recommendations do not transfer across all cooperation situations [9,20], and the needs of car and truck drivers in cooperation situations differ significantly [20], this study aims to clarify the extent to which explanations for system failures are useful in cooperative truck overtaking maneuvers on freeways, and whether they have an impact on trust and acceptance towards the assistance system.

\section{Research Approach}

The purpose of this work is twofold. The first objective is to develop an HMI that enables a high success rate of cooperative truck overtaking maneuvers on freeways. How the design of the HMI can achieve a high usability and thus a high acceptance rate among truck drivers is investigated. The following research questions are formulated.

- RQ1: How can an HMI for cooperative truck overtaking maneuvers on freeways be designed to achieve high usability and thus high cooperation rates?

The second objective of this work is to investigate driving behavior as well as the perceived criticality of system failure during cooperative truck overtaking maneuvers on freeways. System failure is defined as scenarios in which the ADAS stops, either due to technical problems ("system collapse"), e.g., connection or sensor failure, or due to changed traffic and cooperation conditions ("Surrounding traffic does not behave as planned"), e.g., a vehicle that is not equipped with such an ADAS disrupts the cooperation or "the cooperation partner does not behave as planned". Furthermore, the appropriate information content of an HMI in the above-described situations is investigated.

- RQ2: How does system failure influence the driver's behavior?

- RQ3: How do truck drivers evaluate the experienced system failure in terms of criticality?

- RQ4: What information content do truck drivers prefer in the case of a system failure?

\section{Human-Machine Interface}

\subsection{HMI Concept}

The HMI concept consists of a graphical user interface (GUI) shown in the truck's instrument cluster and operated by the truck driver using the multifunction steering wheel. The graphical elements and their arrangement were based on a previously developed HMI (see Figure 1) [9]. It was designed in a user-centered development process [21] based on an extensive analysis of user context and requirements $[6,20]$, and evaluated in two iteration 
loops [9]. The development of the HMI concept in this work constitutes the third iteration loop in this development process.

Graphics are displayed to the truck driver in the middle of the speedometer if the system recognizes potential for cooperation, determined by a vehicle in close proximity ahead and also behind. If the truck driver cooperates with one of the vehicles, it is indicated by a green background and the display of relevant metrics, e.g., the distance and speed of the vehicle [9]. All information related to the cooperation negotiation (e.g., overtaking point found, send request, waiting for confirmation, etc.) is shown in a step-progress bar on the right side of the GUI. The intention is to provide a good overview in the dashboard area and to focus the driver's attention on a few specific subject areas [22]. The numbered sequence of information in the step-progress bar was reduced to a minimum. Compared to the original HMI [9], distracting animated icons were omitted and were replaced with stationary icons (e.g., sand clock) (see Figure 1). In addition to visual information, acoustic information provided by earcons was added [23] to indicate situational changes (e.g., overtaking time point found) [24,25].
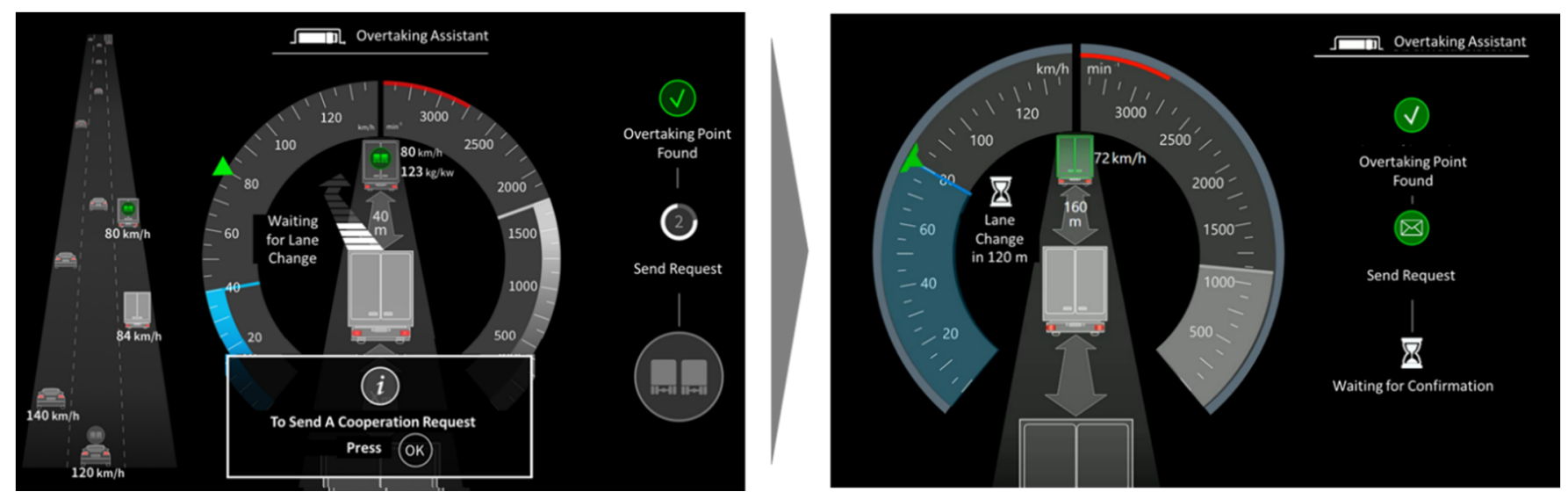

Figure 1. The HMI concept (right) in comparison to the HMI (according to [9]) (left) (figure shows all information simultaneously).

In order to investigate to what extent an automated submission of the cooperation request leads to an increase in the success rate of cooperative truck overtaking maneuvers on freeways, two interaction concepts were designed. The first concept asks the truck driver in the step-progress bar whether a cooperation request should be submitted. Compared to the original HMI [9], the duration of the question was increased from 6 to $10 \mathrm{~s}$ to ensure sufficient transition time to process the content and react accordingly [26,27]. The second concept features an automatic cooperation request, the system steps are also displayed in the step progress bar.

\subsection{Adding Information about System Failures}

In order to reduce the visual stimulus and information affecting the driver when adding abort conditions to the HMI, all abort reasons or indications were displayed exclusively in the step-progress bar (see Figure 2). Symbols that were already used in other driver assistance systems were used so that the driver could quickly comprehend the new situation [28]. In addition, the symbols were chosen unambiguously so that the driver was aware of the system's performance [19]. An icon in the form of two trucks on a red background for "cooperation aborted" (Figure 2) was implemented. A recurrent icon is intended to safeguard the driver's ability to learn [28]. Therefore, a specific icon is always the first indication that the driver receives about the system status when the maneuver is aborted. Initially, it is important to draw the driver's attention to the cancellation itself and, in a following step, to point out the respective reason [29]. 

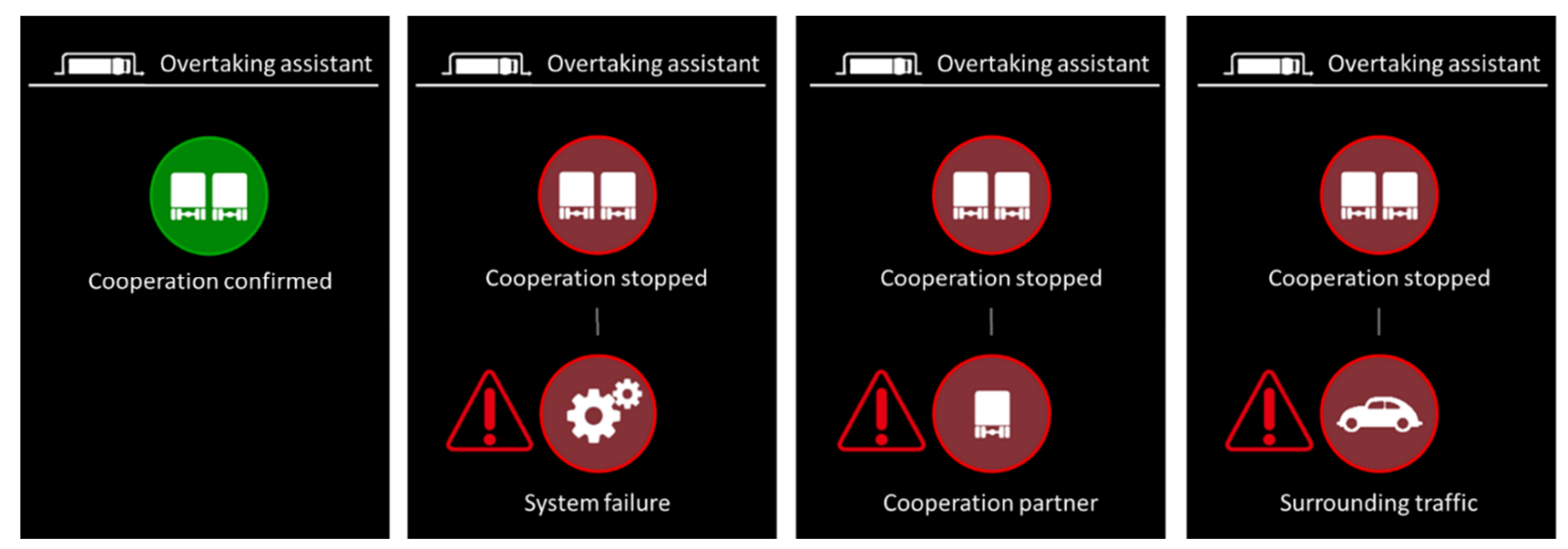

Figure 2. Symbols of the three different systems with explanation text.

In addition to illustrations of the reason for cancellation, a red triangle with a red exclamation mark in the center was displayed to draw the driver's attention to the information. Expectation conformity (cf. Principles of Dialog Design [28]) was increased by using this symbol, since it is well established by the 1968 Vienna Convention on road signs and signals as an indication of an unspecified danger. The symbol was already well known to drivers. According to Shneiderman's third interaction design rule [19], it should be noted that the driver receives informative feedback, which is implemented by newly developed icons or icons and text. If the driver is informed in more detail about the reasons for a failed overtaking maneuver, the feeling of control also increases [19]. The purpose of this study was to investigate whether the display of icons is sufficient or whether additional explanation text is required.

\section{Driving Simulator Study}

\subsection{Hypotheses}

The study was divided into two parts. The first part was dedicated to answering RQ1. We investigate whether the HMI concept achieves a high usability and thus a high success rate of cooperative truck overtaking maneuvers on freeways. Furthermore, the impact of different request initiations on usability and mental load from the perspective of the overtaking driver were considered. For this purpose, two interaction concepts were designed. In the first concept, the drivers themselves submit the request for cooperation (manual) and thus initiate the cooperative maneuver. In the second concept, the ADAS submits the request (automatic). Two hypotheses were formulated:

- Hypothesis 1 (H1). The perceived usability is higher with an automated cooperation request than with a manual cooperation request.

- Hypothesis 2 (H2). The mental load of truck drivers is higher for a manual cooperation request than for an automated request.

Part two of the study investigated the behavior of truck drivers during system failures from the overtaker's perspective. The perceived criticality as well as the subjects' acceptance of and trust in the system were surveyed. For this purpose, two concepts were compared with each other. The first concept tries to explain the underlying reasons for a system failure to the truck driver using only icons (without explanations). The second concept provides the driver with an additional explanatory text (with explanation). Four hypotheses were formulated:

- Hypothesis 3 (H3). System failures are perceived less critically by drivers when explanation text is offered alongside symbols.

- Hypothesis $4 \mathbf{( H 4 ) . ~ T h e ~ s i t u a t i o n a l ~ a w a r e n e s s ~ o f ~ d r i v e r s ~ d u r i n g ~ s y s t e m ~ f a i l u r e s ~ i s ~ h i g h e r ~ i f ~}$ the system provides explanation text in addition to symbols. 
- Hypothesis 5 (H5). Drivers' trust in the system is increased if the system offers explanation text in addition to symbols when system failures occur.

- Hypothesis 6 (H6). Driver acceptance increases if the system offers an explanation in addition to symbols for system failures.

\subsection{Method}

\subsubsection{Participants}

A total of 31 subjects participated in the study. Due to technical problems, one subject had to be excluded from further consideration. The 30 subjects were between 28 and 70 years old $(M=52.4, S D=10.5)$. In total, 28 of them were male. On average, the test subjects had been driving trucks for 27.9 years $(S D=11.09)$. A total of $43.3 \%$ are currently full-time truck drivers, $36.7 \%$ are part-time truck drivers. The remaining $20 \%$ used to work full-time. A total of $46.7 \%$ of the subjects drove more than $150 \mathrm{~km}$ per day (international and national long-distance traffic), $6.7 \%$ of the subjects drove $50-150 \mathrm{~km}$ (regional traffic), and $36.7 \%$ drove up to $50 \mathrm{~km}$ per day (local traffic). Truck drivers were recruited from the data bank available to the institute. The truck drivers received a compensation of EUR 10 per hour.

\subsubsection{Apparatus}

The study was carried out using a dynamic truck driving simulator (see Figure 3). This consisted of an MAN TGS truck cab mounted on a hexa-pod. Due to the 6 degrees of freedom, movements in the driving dynamics could be realized realistically. The simulator had a cylindrical screen that, with the help of 5 projectors, provided the driver with a 210 panoramic view. The rear-view mirrors were represented by 3 LCD monitors. The HMI was shown on a freely programmable display in the position of the original instrument cluster. The driving simulation ran within a computer network with 13 desktop computers. SILAB $^{\odot} 5$ of the Würzburg Institute for Traffic Sciences $\mathrm{GmbH}$ was used as the driving simulator software.

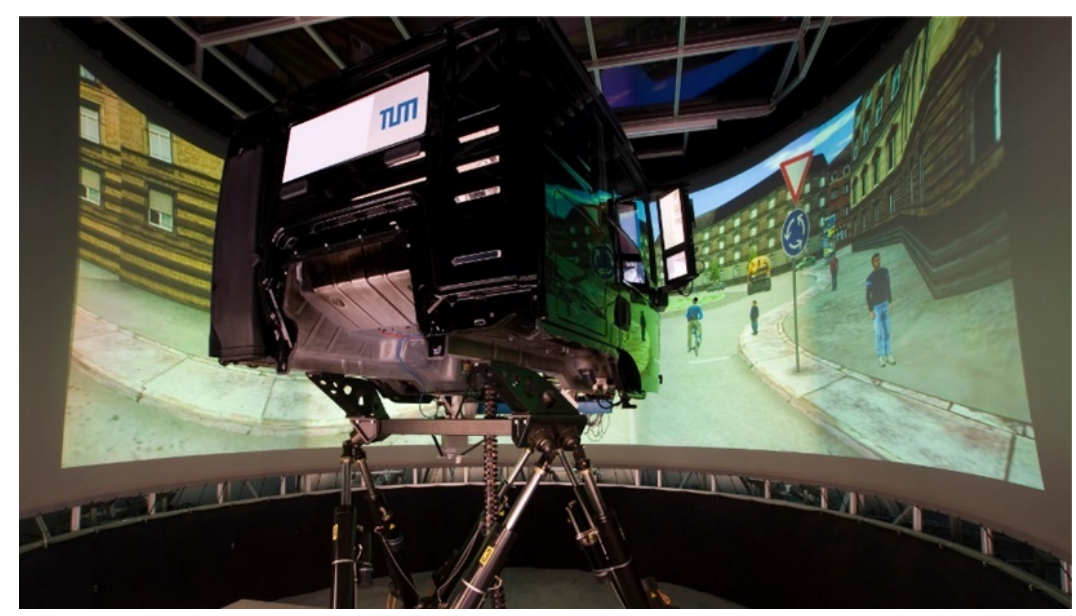

Figure 3. Dynamic truck driving simulator.

\subsubsection{Study Design}

The study was divided into two sections. In the first section, truck drivers evaluated two HMI concepts. In the first concept, truck drivers had to manually send a cooperation request when overtaking. In the second concept, the cooperation request was sent by the system. The sequence of concepts that were experienced was randomized for each truck driver. In the second section, the truck drivers evaluated two HMI concepts for support in the event of the system's failure. The first concept displayed symbols to the truck drivers to explain system errors (see Section 4.2), the second concept displayed explanation text for the system errors in addition to the symbols. Likewise, in the second part, the order of 
the concepts experienced was randomized for each truck driver. In terms of within-subject design, truck drivers experienced both HMI concepts in each of the two study sections.

\subsubsection{Scenario Design}

The simulated track corresponded to a German three-lane highway under good weather conditions. For the first part of the study, a route was designed in which the truck driver overtook another truck twice with a differential speed of $2 \mathrm{~km} / \mathrm{h}$. For the second part, a route was designed in which four overtaking scenarios were implemented:

- $\quad$ S.1 No System Failure: The truck driver overtakes a truck with a $2 \mathrm{~km} / \mathrm{h}$ differential speed without system failure.

- S.2 System Collapse: The truck driver overtakes a truck with $2 \mathrm{~km} / \mathrm{h}$ differential speed. After the trucks are almost at the same level, the system fails, causing the cooperator to accelerate back to the initial speed.

- S.3 Cooperation partner does not behave as planned: The truck driver overtakes a truck with a $2 \mathrm{~km} / \mathrm{h}$ differential speed. Shortly after the overtaker has pulled out, the cooperation partner does not keep to the agreed speed reduction $(-2 \mathrm{~km} / \mathrm{h})$ and accelerates again to the initial speed.

- S.4 Surrounding traffic does not behave as planned: The surrounding traffic does not create a gap for the overtaker to pull out.

Figure 4 shows the moment when the truck driver receives feedback of the system error.

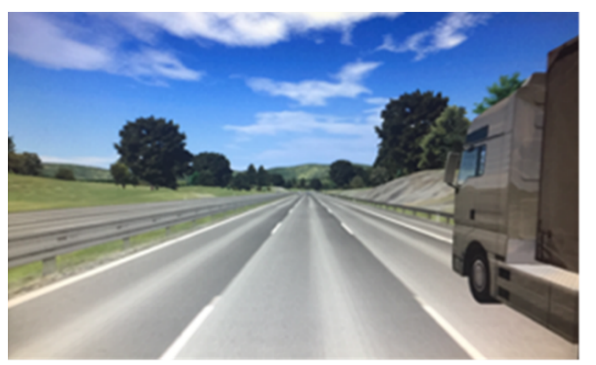

S.2: System collapse

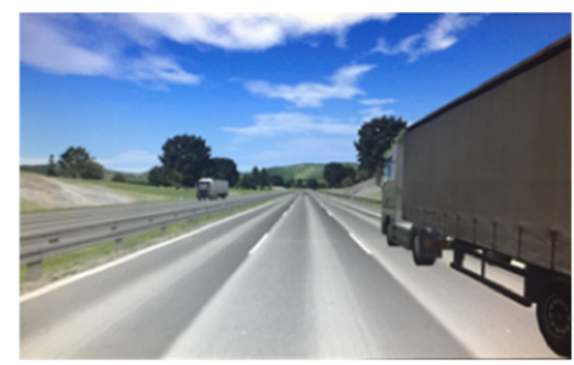

S.3: Cooperation partner does not behave as planned

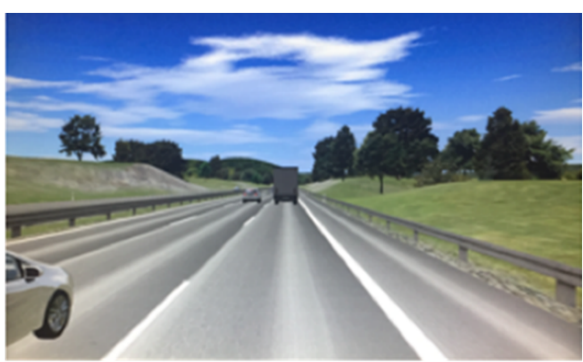

S.4: Surrounding traffic does not behave as planned

Figure 4. Timing of the abort situations S2-S4 from the truck driver's perspective.

\subsubsection{Data Collection}

In addition to the basic driving data (speed, acceleration, etc.), the behavior of the truck drivers was recorded. In the first section of the study, the reasons for unsuccessful overtaking maneuvers were registered. In the second section, the response to system failures was assessed.

In addition to the objective data, usability was also determined in the first section using the System Usability Scale [30], and mental load was determined using the NASATLX questionnaire [31]. After that, they were interviewed on which system they would prefer to use and for what reason. In the second section, the truck drivers were asked about criticality after each driving situation [32]. In addition, they were interviewed after each situation to see if they had any understanding of the type of error that had occurred. At the end of the trip, they were asked about their trust in the system with the help of the trust scale [33] and their acceptance of the system with the Van der Laan questionnaire [34] for the respective HMI. 


\subsubsection{Procedure}

After the truck drivers received an introduction to the driving simulator and the study, they were informed about their personal rights and personal data protection laws. This was followed by a presentation about the cooperative assistance system, as well as the collection of demographic data. After a familiarization drive, the first part of the study began. Truck drivers were randomized to complete a drive with two passing maneuvers and a manual dispatch of cooperative requests, followed by a drive with an automated dispatch of cooperative requests. After each trip, subjective data were collected via a questionnaire. After a short break, the second part of the study began. The drivers completed one trip with four overtaking maneuvers, including abort situations with an HMI that only provided feedback on the abort situation via icon, and one trip with an HMI that provided additional explanation text of the abort conditions. After each abort scenario, the drivers were asked to briefly describe the reason why the maneuver was aborted and how critical they felt the scenario was on a scale from 0 to 9 [32].

\section{Results}

\subsection{Cooperation Request \\ 6.1.1. Driving Behavior}

Table 2 summarizes the number of incidents during the trips with manually and automatically sent cooperation requests. If the cooperation request was sent automatically, cooperative overtaking maneuvers occurred more frequently in $4 \%$ of the cases. However, this difference was not significant $(\mathrm{z}=-0.367, p>0.05, n=30)$.

Table 2. Summary of reasons for unsuccessful overtaking maneuvers.

\begin{tabular}{ccc}
\hline & \multicolumn{2}{c}{ Cooperation Request } \\
\hline & Manual $(\boldsymbol{n = 6 0 )}$ & Automatic $(\boldsymbol{n}=\mathbf{6 0})$ \\
\hline Successful cooperation & $43(71 \%)$ & $45(75 \%)$ \\
\hline Send no request & $10(17 \%)$ & - \\
\hline Lane change too early & $7(12 \%)$ & $15(25 \%)$ \\
\hline
\end{tabular}

\subsubsection{Usability, Mental Workload, Intention to Use}

The HMI with automated sending of cooperation requests received a higher SUS score (with a range from 0 to 100) than the HMI with manual activation (automated: $M=89$, $S D=10$; manual: $M=84, S D=14)$. The Wilcoxon test was used to calculate significance. The analysis shows that the two HMIs differed with a medium effect size $(z=-2.54, p=0.011$; $r=0.46$; mean effect according to Cohen [35]). The examination of mental load using the NASA-TLX (with a range from 0 to 100) revealed no differences (automated: $M=17.9$, $S D=17.1$; manual: $M=19.3, S D=17.0 ; z=-0.56, p=0.798$ ). In the subsequent survey, $83.3 \%$ would prefer an automated cooperation request.

\subsection{System Failure}

\subsubsection{Driving Behavior}

As soon as an abort situation occurred, the respective reaction by the driver was observed. The reaction between the trips with and without explanation text did not change. Therefore, for further consideration, the trips with and without explanation text are summarized for each abort scenario (see Table 3). 
Table 3. Summary of respective reactions as soon as an abort situation occurred.

\begin{tabular}{ccccc}
\hline & No Overtaking & $\begin{array}{c}\text { Overtaking } \\
\text { by Adjusting Speed }\end{array}$ & $\begin{array}{c}\text { Overtaking } \\
\text { with No Reaction }\end{array}$ & Others \\
\hline S.2 System collapse $(n=60)$ & 1 & 1 & 54 & 4 \\
\hline S.3 Cooperation partner $(n=60)$ & 33 & 9 & 12 & 6 \\
\hline S.4 Surrounding traffic $(n=60)$ & 56 & 2 & - & 2 \\
\hline
\end{tabular}

In the incident with "system collapse" (S.2) as the reason, truck drivers continued the overtaking maneuver without assistance in approximately $90 \%$ of the cases. Only about $2 \%$ of drivers aborted the maneuver by braking and merging back into the right lane. In the incident "Cooperation partner does not behave as expected" (S.3), the truck drivers aborted the overtaking maneuver in about $50 \%$ of the cases by braking and merging back into the right lane behind the slower truck. In about $20 \%$ of the cases, the truck drivers stayed in the left lane. In approximately $15 \%$ of the cases, the truck drivers increased their speed to overtake anyway. In the "surrounding traffic not behaving as planned" incident (S.4), truck drivers did not overtake in approximately $92 \%$ of the cases.

\subsubsection{Criticality, Situational Awareness, Trust, and Acceptance}

After each drive with and without system failure, the subjects were asked to rate the criticality of the driving situation they had just experienced. Figure 5 illustrates the results for all four simulated situations (S.1 = no incident, $\mathrm{S} .2=$ system collapse, $\mathrm{S} .3=$ cooperation partner, and S.4 = surrounding traffic). No significant difference was found in the criticality rating between the respective situations with and without explanation (Table 4). The "cooperation partner does not behave as planned" abort scenario (S.3) was rated most critically by the truck drivers.

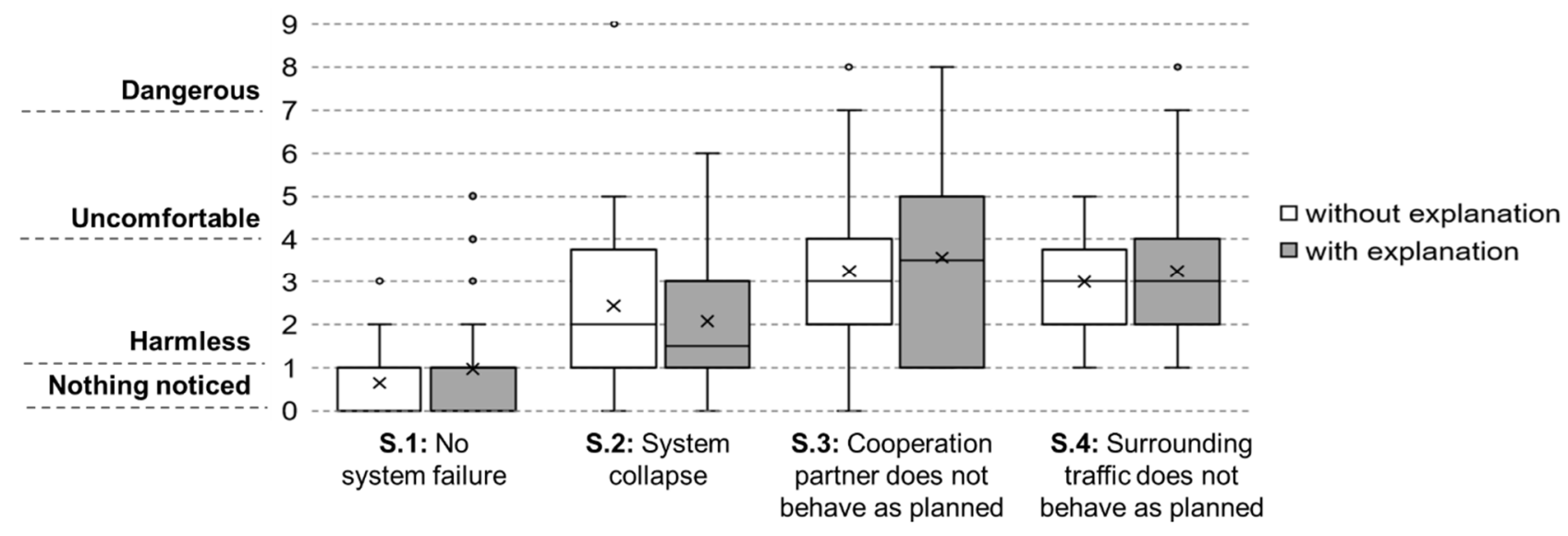

Figure 5. Perceived criticality in the individual route situations in drives with and without explanation text for system failures.

From the driver's memory, we can deduce information about their situational awareness. For each HMI, $n=90$ trips were analyzed. The situational awareness of system failures with explanation text was higher $(91.1 \%)$ in comparison to the situation without explanation text $(85.6 \%)$. However, this difference was not significant (Table 4 ).

System trust was measured with the subjective questionnaire trust scale [33]. This consisted of 12 question items that were averaged for each HMI. No significant difference was calculated between the two scores (with and without explanation).

To evaluate acceptability, Van der Laan's subjective questionnaire [34] was used. It divides acceptance into a usefulness score and a satisfaction score. These are each in the 
range of -2 to +2 , with +2 being the best value to be achieved. Neither the usefulness score or the satisfaction score showed a significant difference.

Table 4. Descriptive and inferential statistics of the evaluation of HMI with and without explanation.

\begin{tabular}{|c|c|c|c|}
\hline & Without $M(S D)$ & With $M(S D)$ & Interference Statistic \\
\hline \multicolumn{4}{|l|}{ Criticality (scale from 0 to 9 ) } \\
\hline $\mathrm{S} .1^{1}(n=30)$ & $0.64(0.87)$ & $0.96(1.32)$ & $\mathrm{z}=-1.19, p=0.23$ \\
\hline $\mathrm{S} .2(n=30)$ & $2.46(2.10)$ & $2.07(1.44)$ & $z=-1.56, p=0.12$ \\
\hline S.3 $(n=30)$ & $3.25(1.99)$ & $3.57(2.28)$ & $\mathrm{z}=-0.81, p=0.42$ \\
\hline $\mathrm{S} .4(n=30)$ & $3.00(1.59)$ & $3.25(1.59)$ & $\mathrm{z}=-0.77, p=0.44$ \\
\hline $\begin{array}{l}\text { Situational awareness }(n=90) \\
\text { (number of correct answers) }\end{array}$ & 77 & 82 & $\mathrm{X}^{2}(1, n=30)=0.43, p=0.51$ \\
\hline Trust $(n=30)($ scale from 0 to 5$)$ & $4.76(0.74)$ & $4.79(0.78)$ & $\mathrm{z}=-0.53, p=0.60$ \\
\hline \multicolumn{4}{|l|}{ Acceptance (scale from -2 to 2) } \\
\hline Usefulness $(n=30)$ & $1.18(0.69)$ & $1.25(0.65)$ & $\mathrm{z}=-0.85, p=0.39$ \\
\hline Satisfaction $(n=30)$ & $0.93(0.85)$ & $1.06(0.69)$ & $\mathrm{z}=-1.42, p=0.16$ \\
\hline
\end{tabular}

${ }^{1}$ S.1 = without system failure; S.2 = system collapse; S.3 = cooperation partner does not behave as planned; S.4 = surrounding traffic does not behave as planned.

\section{Discussion}

The goal of this work was to design an HMI concept that enables a high cooperation rate in truck overtaking maneuvers on freeways. The assumption was to achieve a high usability of the underlying ADAS system. In addition, this work aimed to analyze the driving behavior of truck drivers in the case of system failure during truck overtaking maneuvers on freeways, and to measure their confidence and acceptance towards the system as well as the perceived criticality of the individual system failures. For this purpose, a two-part concept was developed to answer four research questions and six hypotheses, which are discussed in this section:

RQ1: How can an HMI for cooperative truck overtaking maneuvers on freeways be designed to achieve high usability and thus high cooperation rates? To achieve high usability, an HMI concept based on an existing HMI for cooperative truck overtaking maneuvers on freeways [9] was developed considering established design paradigms. According to [36], the subjective usability values (automatic: 89 and manual: 84 ) are in an "excellent" range. This shows that the HMI concept has accomplished a high level of usability. In the analysis of successful cooperation maneuvers, a high value of approximately $75 \%$ was achieved. However, in $25 \%$ of the cases there were still erroneous overtaking maneuvers because truck drivers changed their lanes too early or too late. In a further evaluation, the instruction to change lanes could be improved again. With a usability score of over 80 points, it is not clear whether the remaining $25 \%$ could be achieved with further improvement in the domain of usability. The integration of motivational strategies could be investigated for the potential to increase the success rate. In previous studies in which car drivers were motivated to cooperate by means of gamification, encouraging results could already be achieved [37]. Another way to increase the willingness to cooperate would be to actively convince the truck driver to only execute the lane change when instructed to do so by the system. It is already known from human-robot interaction that the presence of a human assistant such as an avatar or robot can increase the persuasive power of the assistant [38,39]. Subjects are thus inclined towards doing things they did not initially intend to do. Initial studies on the presence of a humanized assistant in a truck have also shown positive effects $[14,40]$. In a separate approach, the idea of shared control concepts, e.g., H-metaphor [41,42], could be used to increase the success rate of cooperative overtaking maneuvers of truck on freeways. This would introduce a new set of paradigms for the HMI design and might require an entirely new development process. 
In addition to the general answer to RQ1, the assumption was also made that an automated cooperation request can increase the cooperation rate, since usability increases and the mental load remains the same. The answers to the associated hypotheses are discussed below:

Hypothesis 1 (H1). Usability is higher with an automated cooperation request. The results show that, compared to manual submitting, automatic submitting of cooperation requests achieves a significant improvement in usability with a medium effect. However, this is not translated into an increased cooperation rate. The difference between the number of successful cooperation maneuvers with automated request and manual request is not significant. The majority of truck drivers would agree to an automated request, however, when being interviewed, the truck drivers expressed their desire to be able to veto and withdraw the cooperation request $(N=5)$.

Hypothesis 2 (H2). Mental workload is lower with an automated cooperation request. There was no significant difference between the tested variants with regard to the subjective mental workload. Therefore, possible incorrect operation of the system cannot be attributed to a change in mental workload.

RQ2: How does system failure influence the driver's behavior? The results show that the behavior of the drivers varies depending on the type of system failure. When unplanned behavior of the surrounding traffic occurs before the overtaking maneuver, most subjects aborted the overtaking maneuver. If a system failure occurs during the overtaking maneuver, most subjects still continue to perform the overtaking maneuver. If the person being overtaken behaves in an unexpected pattern during the overtaking maneuver, half of the subjects aborted the overtaking maneuver. Here, it is reasonable to assume that the subjects' behavior depends on the progress of the overtaking maneuver, since the abort rate decreases with a later occurrence of failure in the overtaking maneuver. Hence, the consequences of system failures are that either no overtaking maneuver takes place or that the overtaking maneuver is prolonged, which is what the system initially aims to avoid. Risky maneuvers or even accidents are therefore not expected.

RQ3: How do truck drivers evaluate the experienced system failure in terms of criticality? Criticality was rated in the low to medium range. A system collapse was rated as "harmless", an unplanned behavior of the cooperating partner or the surrounding traffic was perceived as "unpleasant". Since the subjects did not rate any of the induced situations as dangerous or uncontrollable, system failures during truck overtaking maneuvers were not rated as safety-critical. The results are thus similar to those in studies of cooperative maneuvers with passenger car drivers [15]. These perceived system failures during cooperative left-turn maneuvers or cooperative maneuvers on freeway ramps ranged from being harmless to unpleasant as well.

RQ4: What information content do truck drivers prefer in the case of a system failure? Two HMI concepts were implemented that differed in the degree of displayed information content. One HMI showed feedback to the driver via icons, while the other provided additional explanations in text form. To answer RQ4, the influence of the level of information on the situational awareness, trust, and acceptance of the truck drivers was investigated. In the following, the related hypotheses $\mathrm{H} 3-\mathrm{H} 6$ will be answered:

Hypothesis 3 (H3). System failures are perceived less critically by drivers when explanation text is offered alongside symbols. The criticality perceived for system failures was felt to be the same for both HMI variants. The criticality is in the low to medium range for both concepts.

Hypothesis 4 (H4). The situational awareness of drivers during system failures is higher if the system provides explanation text in addition to symbols. The abort situation was not significantly incorrectly recognized when only a symbol for the system failure was displayed in comparison to the text-enhanced version. This suggests that the symbols are sufficient and unambiguous for explanation [21]. 
Hypothesis 5 (H5). Drivers' trust in the system is increased if the system offers explanation text in addition to symbols when system failures occur. There were no significant differences between the two HMI concepts in the truck drivers' evaluation of trust in the system. An additional explanation in text form was therefore not perceived as more trustworthy. With a subjective value of approximately 4.75 out of a maximum of 5 points, both HMI variants revealed a high level of trust by the truck drivers towards the system, despite the occurrence of system failures.

Hypothesis 6 (H6). Driver acceptance increases if the system offers an explanation in addition to symbols for system failures. Acceptance of the system was also not increased by additional explanation of system failures. Here, too, the achieved scores are in the acceptable range [34].

\section{Limitations}

In the evaluation of the ADAS concept with regard to the success rate of cooperative truck overtaking maneuvers on freeways, the subjectively perceived usability, mental stress, and the objective evaluation of driving behavior were used as evaluation parameters. To conclusively measure the quality of the system, it is certainly necessary to consider other metrics, such as the drivers' reaction time to individual HMI elements or their gaze behavior.

The overall results suggest that truck drivers see the system for cooperative truck overtaking maneuvers on freeways as supportive and helpful for the recognition of cooperative partners and for the engagement in a cooperative maneuver. System failures as simulated are considered non-critical, and thus have little impact on the trust in and acceptance of the system. However, it should be noted that system failures always occurred at the same time. In future investigations, the occurrence of the errors should be varied and tested in addition to other system failures.

The number of possible system failures in the case of cooperative truck overtaking maneuvers was reduced to three cases for operational reasons. However, in the use of V2X communication, other system failures are certainly to be expected, e.g., trajectories transmitted incorrectly, etc. In addition, traffic is a highly dynamic environment. In this setting, the only deviation examined was when the surrounding traffic does not adjust the speed as desired for an overtaking maneuver. However, we did not investigate how critical the truck drivers consider it when lane changes are performed by surrounding traffic during a cooperative maneuver, e.g., a car without ADAS pulls in shortly in front of a truck to exit the highway. These and other maneuvers certainly need further investigation.

Furthermore, final conclusions on the effects of system failures on truck drivers during cooperative truck overtaking maneuvers can only be drawn if real vehicle studies are used in addition to driving simulator studies. It is possible that system failures can be evaluated more critically in a real vehicle than in a driving simulation. It cannot be dismissed that the truck drivers in the protected environment of the test setup perceived the system failures less critically than under real conditions.

\section{Conclusions}

In this work, an HMI for cooperative truck overtaking maneuvers on freeways was evaluated and extended to include warnings about system failures. In a driving simulation study with 30 truck drivers, the HMI concept was analyzed with regards to usability, mental load, trust, and acceptance. Usability was improved by reducing the information content, adding acoustic signals, and repositioning used icons. In addition, the implemented automatic dispatch of cooperation requests reduced operator errors without affecting their mental load. Despite the reduction in operator errors, truck drivers changed lanes too early in $25 \%$ of the cases. Further studies should investigate whether the success rate of cooperative truck overtaking maneuvers can be further increased by motivational and persuasion strategies. The evaluation of possible display concepts for system failures found that icons are sufficient in this regard and no additional explanations in text form are required. Despite simulated system failures, the assistance system achieved high 
confidence and acceptance scores. This can be explained by the fact that the criticality of the system errors was perceived as only harmless to unpleasant. The system errors resulted in either no overtaking maneuver or in the overtaking maneuver being dragged out. No critical driving maneuvers or accidents occurred. These results should be verified in future real vehicle studies. Furthermore, additional system failures and the timing of their varying occurrences should be evaluated.

Author Contributions: Conceptualization, J.F. and C.K.; methodology, J.F. and C.K.; software, J.F. and C.K.; validation, J.F. and C.K.; writing—original draft preparation, J.F.; writing—review and editing, J.F., C.K., and F.D.; visualization, J.F.; supervision, F.D.; project administration, F.D.; funding acquisition, F.D. All authors have read and agreed to the published version of the manuscript.

Funding: This work was supported by the German Federal Ministry for Economic Affairs and Energy within the research project IMAGinE (Intelligent Maneuver Automation-cooperative hazard avoidance in real time).

Institutional Review Board Statement: The study was conducted according to the guidelines of the Declaration of Helsinki. Ethical review and approval were waived for this study as no ethical issues were involved (e.g., no vulnerable populations, no collection of sensitive issues, no distressing situations, invasive activities, or collection of biological materials).

Informed Consent Statement: Informed consent was obtained from all subjects involved in the study.

Acknowledgments: Special thanks go to Katharina Els, Fabian Schlichtherle, and Yunzhi Zhou who contributed to the design and implementation of the HMI as well as to the design of the study and evaluation, and Pascal Hoffmann, who contributed to the design of the cooperative function, study, and evaluation.

Conflicts of Interest: The authors declare no conflict of interest.

\section{References}

1. Fekete, S.; Vollrath, M.; Huemer, A.K.; Salchow, C. Interaktion im Straßenverkehr: Kooperation und 670 Konflikt. In Proceedings of the 8. VDI-Tagung Der Fahrer im 21. Jahrhundert, Fahrer, Fahrunterstützung und Bedienbarkeit, Brunswick, Germany, 10-11 November 2015; pp. 325-671.

2. Mertens, J.C.; Knies, C.; Diermeyer, F.; Escherle, S.; Kraus, S. The Need for Cooperative Automated Driving. Electronics 2020, 9 , 754. [CrossRef]

3. Knies, C.; Fank, J.; Diermeyer, F. How to Measure Cooperation? Cost Functions for Cooperative Maneuver Planning on Highways. In Proceedings of the IEEE Intelligent Transportation Systems Conference (ITSC), Auckland, New Zealand, 27-30 October 2019; pp. 2483-2489. [CrossRef]

4. Kraft, A.-K.; Maag, C.; Bauman, M. How to support cooperative driving by HMI design? Transp. Res. Interdiscip. Perspect. 2019, 3, 100064. [CrossRef]

5. Ellinghaus, D.; Streinbrecher, J. Lkw im Straßenverkehr: Eine Untersuchung über die Beziehung zwischen Lkw- und Pkw-Fahrern. UNIROYAL-Verkehrsuntersuchung 2002, 27, $247 \mathrm{~S}$.

6. Fank, J.; Krebs, P.; Diemreyer, F. Analyse von Lkw-Überholmanövern auf Autobahnen für die Entwicklung kooperativer Fahrerassistenzsysteme. Ingenieurwes 2019, 83, 305-316. [CrossRef]

7. Mertens, J.C.; Hauenstein, J.; Diermeyer, F.; Jahn, L.; Kraus, S. Cooperative Truck Overtaking on Freeways. In Proceedings of the Fifteenth International Conference on Ecological Vehicles and Renewable Energies (EVER) 2020, Monte-Carlo, Monaco, 10-12 September 2020; pp. 1-15. [CrossRef]

8. Mertens, J.C.; Hauenstein, J.; Diermeyer, F.; Zimmermann, A. Strategic Coordination of Cooperative Truck Overtaking Maneuvers. In Proceedings of the 7th International Conference on Vehicle Technology and Intelligent Transport Systems: VEHITS 2021, Prague, Czech Republic, 28-30 April 2021; Volume 1, pp. 513-520. [CrossRef]

9. Fank, J.; Knies, C.; Diermeyer, F. After You! Design and Evaluation of a Human Machine Interface for Cooperative Truck Overtaking Maneuvers on Freeways. In Proceedings of the 13th International Conference on Automotive User Interfaces and Interactive Vehicular Applications (AutomotiveUI '21), Leeds, UK, 9-14 September 2021. [CrossRef]

10. Kraft, A.K.; Maag, C.; Cruz, M.I.; Baumann, M.; Neukum, A. The effect of visual HMIs of a system assisting manual drivers in manoeuvre coordination in system limit and system failure situations. Transp. Res. Part F Traffic Psychol. Behav. 2020, 74, 81-94. [CrossRef] 
11. Davis, D. A Technology Acceptance Model for Empirically Testing New End-User Information Systems-Theory and Results. Ph.D. Thesis, Massachusetts Institute of Technology, Cambridge, MA, USA, 1885.

12. Endsley, M.R. Situation Awareness. In Handbook of Human Factors and Ergonomics; John Wiley \& Sons: Hoboken, NJ, USA, 2006; pp. 528-542. [CrossRef]

13. Zimmermann, M. Cooperative Interaction. Within Cars and Between Drivers. Ph.D. Thesis, Faculty Mechanical Engineering, Technical University Munich, Munich, Germany, 2018.

14. Fank, J.; Santen, L.; Knies, C.; Diermeyer, F. Should We Allow Him to Pass? Increasing Cooperation between Truck Drivers Using Anthropomorphism. In Advances in Human Factors of Transportation (AHFE); Springer: Cham, Switzerland, 2019; Volume 964, pp. 475-484. [CrossRef]

15. Kraft, A.K.; Maag, C.; Cruz, M.I.; Baumann, M.; Neukum, A. Effects of explaining system failures during maneuver coordination while driving manual or automated. Accid. Anal. Prev. 2020, 148, 105839. [CrossRef] [PubMed]

16. SAE. Taxonomy and Definitions for Terms Related to On-Road Motor Vehicle Automated Driving Systems. SAE Stand. J. 2018, 3016, 1-6.

17. Lee, J.D.; See, K.A. Trust in automation: Designing for appropriate reliance. Hum. Factors 2014, 46, 50-80. [CrossRef] [PubMed]

18. Verberne, F.; Ham, J.; Midden, C. Trust in smart systems sharing driving goals and giving information to increase trustworthiness and acceptability of smart systems in cars. Hum. Factors J. Hum. Factors Ergon. Soc. 2012, 54, 799-810. [CrossRef] [PubMed]

19. Shneiderman, B. Designing the User Interface: Strategies for Effective Human-Computer Interaction, 3rd ed.; Addison-Wesley Longman Publishing Co. Inc.: Hoboken, NJ, USA, 1997.

20. Fank, J.; Knies, C.; Diermeyer, F.; Prasch, L.; Reinhardt, J.; Bengler, K. Factors for User Acceptance of Cooperative Assistance Systems: A Two-Step Study Assessing Cooperative Driving. In Proceedings of the Tagung der Fahrerassistenz, Munich, Germany, 22-23 November 2017; Volume 8.

21. Ergonomics of human-system interaction-Part 210: Human-centred design for interactive systems (ISO 9241-210:2019); German version EN ISO 9241-210:2019. Available online: https://www.beuth.de/en/standard/din-en-iso-9241-210/313017070 (accessed on 29 October 2021).

22. Kim, S.H.; Sabando, J.F.; Kim, W.H. An Ecological Interface Design Approach for Developing Integrated and Advanced In-Vehicle Information System. Indian J. Sci. Technol. 2016, 9, 92038. [CrossRef]

23. Seppelt, B.; Wickens, C.D. Interference with Driving or In-Vehicle Task Information: The Effects of Auditory Versus Visual Delivery; AHFD-02-18/GM-02-3; University of Illinois, Aviation Research Laboratory: Savoy, IL, USA, 2002.

24. Bubb, H. Systemergonomische Gestaltung. In Ergonomie; Schmidtke, H., Ed.; Hanser Verlag: München, Germany, 1993.

25. Lerner, N.D. Preliminary Human Factors Guidelines for Crash Avoidance Warning Devices; NHTSA Project No. DTNH22-91-C-07004; COMSIS Corporation: Silver Spring, MD, USA, 1996.

26. Damböck, D.; Kienle, M.; Bengler, K. Bedienelemente hochautomatisierter Fahrzeuge. Studie zum haptischen Kanal als Kommunikationsmedium. In Neue Arbeits- und Lebenswelten gestalten - 56. Kongress der Gesellschaft für Arbeitsswissenschaften; GfA Press: Darmstadt, Germany, 2010; pp. 157-160.

27. Melcher, V.; Rauh, S.; Diederichs, F.; Widlroither, H.; Bauer, W. Take-Over Requests for Automated Driving. Procedia Manuf. 2015, 3, 2867-2873. [CrossRef]

28. DIN EN ISO 9241-110:2020, Ergonomics of Human-System Interaction-Part 110: Interaction Principles (ISO 9241-110:2020); German Version EN ISO 9241-110:2020. Available online: https:/ / www.beuth.de/en/standard/din-en-iso-9241-110/320862700 (accessed on 29 October 2021).

29. Nielsen, J.; Molich, R. Heuristic evaluation of user interfaces. In Proceedings of the SIGCHI Conference on Human Factors in Computing Systems Empowering People-CHI ‘90, Seattle, Washington, DC, USA, 1-5 April 1990; pp. 249-256.

30. Brooke, J. SUS: A quick and dirty usability scale. Usability Eval. Ind. 1995, 189, 4-7.

31. Hart, S.G. Nasa-Task Load Index (NASA-TLX); 20 Years Later. Proc. Hum. Factors Ergon. Soc. Annu. Meet. 2016, 50, 904-908. [CrossRef]

32. Neukum, A.; Lübbeke, T.; Krüger, H.-P.; Mayser, C.; Steinle, J. ACC-Stop\&Go: Fahrerverhalten an funktionalen Systemgrenzen. In 5. Workshop Fahrerassistenzsysteme, Proceedings of the FAS 2008, Walting im Altmühltal, Germany, 2-4 April 2008; Maurer, M., Ed.; Karlsruhe Fmrt: Walting, Germany, 2008.

33. Jian, J.Y.; Bisantz, A.M.; Drury, C.G. Foundations for an Empirically Determined Scale of Trust in Automated Systems. Int. J. Cogn. Ergon. 2000, 4, 53-71. [CrossRef]

34. Van der Laan, J.D.; Heino, A.; de Waard, D. A simple procedure for the assessment of acceptance of advanced transport telematics. Transp. Res. Part C Emerg. Technol. 1197, 5, 1-10. [CrossRef]

35. Cohen, J. A power primer. Psychol. Bull. 1992, 122, 155-159. [CrossRef]

36. Bangor, A.; Kortum, P.T.; Miller, J.T. An Empirical Evaluation of the System Usability Scale. Int. J. Hum.-Comput. Interact. 2008, 24, 574-594. [CrossRef]

37. Zimmermann, M.; Schopf, D.; Lütteken, N.; Liu, Z.; Storost, K.; Baumann, M.; Happee, R.; Bengler, K.J. Carrot and stick: A game-theoretic approach to motivate cooperative driving through social interaction. Transp. Res. Part C Emerg. Technol. 2018, 88, 159-175. [CrossRef] 
38. Ham, J.; Midden, C. A Persuasive Robotic Agent to Save Energy: The Influence of Social Feedback, Feedback Valence and Task Similarity on Energy Conservation Behavior. In Social Robotics, Proceedings of the ICSR 2010, Singapore, 23-24 November 2010; Ge, S.S., Li, H., Cabibihan, J.J., Tan, Y.K., Eds.; Lecture Notes in Computer Science; Springer: Berlin/Heidelberg, Germany, 2010; Volume 6414.

39. Ham, J.; Cuijpers, R.H.; Cabibihan, J.J. Combining Robotic Persuasive Strategies: The Persuasive Power of a Storytelling Robot that Uses Gazing and Gestures. Int. J. Soc. Robot. 2015, 7, 479-487. [CrossRef]

40. Fank, J.; Diermeyer, F. “Talk to My Face!” Analyzing the Effects of Embodiment in Humanized Human-Machine Interaction in Heavy Trucks. In Proceedings of the 32nd IEEE Intelligent Vehicles Symposium (IV), Nagoya, Japan, 11-15 July 2021.

41. Flemisch, F.; Adams, C.; Conway, S.; Goodrich, K.; Palmer, M.; Schutte, M. The H-Metaphor as a Guideline for Vehicle Automation and Interaction; Rep. NASA/TM-2003-212672; NASA: Langley, VA, USA, 2003.

42. Martínez-García, M.; Kalawsky, R.S.; Gordon, T.; Smith, T.; Meng, Q.; Flemisch, F. Communication and Interaction with Semiautonomous Ground Vehicles by Force Control Steering. IEEE Trans. Cybern. 2021, 51, 3913-3924. [CrossRef] [PubMed] 